\title{
Mindfulness training reduces PTSD symptoms and improves stress-related health outcomes in police officers
}

Daniel W. Grupe ${ }^{1}$, Chad McGehee ${ }^{1}$, Chris Smith$^{2}$, Andrew D. Francis ${ }^{1}$, Jeanette A. Mumford ${ }^{1}$, \& Richard J. Davidson ${ }^{1,3,4}$

1. Center for Healthy Minds, University of Wisconsin-Madison; 2. Academy for Mindfulness, LLC; 3. Department of Psychology, University of Wisconsin-Madison; 4. Department of Psychiatry, University of Wisconsin-Madison

\section{Acknowledgements}

This project was supported by the NIH CTSA at UW-Madison grant UL1TR000427, as well as the University of Wisconsin-Madison School of Medicine and Public Health's Wisconsin Partnership Program, WPP-ICTR grant \# 3086.

The authors thank Robin Goldman, John Koger, Mara Beebe, Cara Edmonson, DK Jang, and the staff of the UW Hospital Clinical Research Unit for contributing to study design and data collection; Lori Gustafson for conducting focus groups; Sophia Diamantis for conducting interviews; Mike Christopher, Lt. Rich Goerling, and Brant Rodgers for providing consultation on the intervention; and members of the Madison Police Department for contributing to this project, including Ofc. Sue Carnell (ret.), Chief Kristen Roman (University of WisconsinMadison Police Department), Capt. Tim Patton, Capt. Tom Snyder (ret.), Capt. Mary Schauf (ret.), Capt. Brian Austin, Ofc. Dan Frei, Chief Mike Koval, and all of the study participants.

\section{Contact Information}

Daniel Grupe

Center for Healthy Minds, University of Wisconsin-Madison

625 West Washington Ave, Madison, WI 53703

grupe@wisc.edu

608-263-7572 


\title{
Mindfulness training reduces PTSD symptoms and improves stress-related health outcomes in police officers
}

\begin{abstract}
There are few empirically supported interventions to proactively address stress-related health outcomes in police officers. Recent studies suggest that training in mindfulness meditation may reduce perceived stress and improve related physical and mental health outcomes in this group. We sought to demonstrate feasibility, acceptability, and adherence for an 8-week mindfulness training program in 30 officers from a mid-sized, Midwestern U.S. police department, replicate findings of improved stress-related health outcomes, and provide novel evidence for reduced PTSD symptoms. All 30 officers completed the training, with high rates of class attendance, substantial out-of-class practice time, and good acceptability of the training and teachers. We replicated findings of reduced post-training perceived stress, sleep disturbances, anxiety, and burnout. We also identified novel evidence for reduced PTSD symptoms that persisted at a 5month follow-up assessment. These results indicate key targets for future investigation in larger, mechanistic, randomized controlled trials of mindfulness training in police officers.
\end{abstract}




\section{Mindfulness training reduces PTSD symptoms and improves stress-related health outcomes in police officers}

\section{Introduction}

The many stressors of police work can have profound consequences for the health and well-being of law enforcement officers (LEOs). Chronic work stress is a major contributor to elevated rates of cardiovascular disease (CVD) and CVD risk factors, including obesity and metabolic syndrome, in current and retired LEOs (Franke, Collins, \& Hinz, 1998; Hartley, Burchfiel, Fekedulegn, Andrew, \& Violanti, 2012; Ramey, Downing, \& Franke, 2009; Zimmerman, 2012). Sleep disturbances are endemic among officers working both day and night shifts (Neylan et al., 2002), with one study finding that $40 \%$ of officers screened positive for a sleep disorder and 30\% reported excessive daytime sleepiness (Rajaratnam et al., 2011). Clinically significant symptoms of depression have been reported in 12-16\% of officers (Hartley et al., 2012; Violanti et al., 2006), and a study of midwestern US police officers found that 25\% screened positive for posttraumatic stress disorder (PTSD) or subthreshold PTSD (Chopko, Palmieri, \& Adams, 2018). Concerns about stigma and perceived weakness, however, prevent many officers from reporting mental health concerns or seeking employer assistance (Fox et al., 2012).

Crucially, challenges to officer well-being are felt not just by these individuals but by the entire community. For example, one study found that officers who screened positive for sleep disorders - which co-occurred with depression, anxiety, and burnout - were more likely to commit errors due to fatigue, express anger toward others, and garner complaints from citizens (Rajaratnam et al., 2011). “Officers who are well”, writes Lt. Rich Goerling of the Hillsboro (OR) Police Department, “will provide better police services; officers who are resilient will 
sustain wellness through acute and chronic trauma ... Communities should demand police officers that are well and resilient" (Goerling, 2012).

Most salient among the stressors of police work are acute, potentially traumatic events (or "critical incidents"), which are experienced by almost $90 \%$ of urban police officers in their first year on the job (Galatzer-Levy et al., 2013). Somewhat surprisingly, more routine aspects of the police work environment - including both operational stressors (e.g., shift work, court cases, working alone in dangerous areas) and organizational stressors of bureaucratic police organizations (e.g., staff shortages, lack of resources, insufficient support from leadership) have been cited by LEOs as a greater source of stress than critical event exposure (Brown \& Campbell, 1990; Gershon, Barocas, Canton, Li, \& Vlahov, 2009; Liberman, Best, Metzler, Fagan, \& Weiss, 2002). Moreover, when routine environmental stress and critical incident exposure have been assessed in the same officers, the stress from officers' daily work environment shows stronger relationships with both sleep disturbances (Neylan et al., 2002) and PTSD symptoms (Maguen et al., 2009) than does critical incident exposure. As such, providing officers with strategies and skills to cope with daily occupational and organizational stressors seems critical for improving stress-related outcomes in this population.

Unfortunately, few evidence-based stress-management interventions have been developed with the occupational and cultural considerations of this population in mind. A 2012 review concluded that what few interventions existed at that time had "no significant effect on psychological, behavioral, or physiological outcomes" (Patterson, Chung, \& Swan, 2012). One important consideration in developing a stress-management intervention for police officers is that it be skill-based and embodied, and not merely didactic in nature, in fitting with the trainingfocused culture of policing. Ideally the skills or techniques offered by such a training could be integrated into work to address daily stressors, and not focused on critical incidents alone or 
divorced from officers' work entirely. Further, it is important to focus on developing strengths and building capacity to work with stressful events, as an intervention that focuses on addressing problems would elicit less engagement from police officers who are hesitant to seek treatment or self-identify as having emotional problems or mental health concerns.

Mindfulness-based interventions (MBIs) may provide a culturally appropriate and effective means of addressing LEO stress and its deleterious consequences. Following up on the broad success of mindfulness-based stress reduction (MBSR), originally developed by Jon Kabat-Zinn for the treatment of chronic pain, a number of MBIs have been developed with specific populations and conditions in mind over the past 30 years. These interventions promote the cultivation of intentional, non-judgmental awareness of thoughts, emotions, and sensations as they arise and dissipate through sitting and walking meditation, movement practices, selfinquiry, and group discussions. MBIs are a good fit for the culture of policing in that they are embodied and skill-based, with practices that can be integrated into the activities of daily living. Further, while one need not be suffering from any particular ailment to develop the skills afforded by MBIs, meta-analyses of MBIs show consistent reductions in perceived stress, anxiety, and depression (Goyal et al., 2014; Khoury et al., 2013; Khoury, Sharma, Rush, \& Fournier, 2015), with some evidence for improved sleep (Black et al., 2015; Surawy, Roberts, \& Silver, 2005) and reductions in inflammatory markers that may confer risk for CVD, depression, and PTSD (Black \& Slavich, 2016).

Working with officers in the metro Portland (OR) region, researchers from Pacific University developed Mindfulness-Based Resilience Training (MBRT), the first MBI tailored specifically for LEOs and other first responders. In a pilot feasibility study (Christopher et al., 2016) and follow-up randomized controlled trial (Christopher et al., 2018), researchers identified reductions in perceived stress, depression, anxiety, disrupted sleep, burnout, and self-reported 
anger, along with alterations to the cortisol awakening response. These early studies provide a starting point for future studies that seek to generalize results to new departments, geographical regions, and instructors, while investigating additional outcomes that may be affected by these interventions. One question of particular interest that has not yet been addressed is whether mindfulness training may reduce symptoms of PTSD in police officers, as has been recently demonstrated in military veterans (Polusny et al., 2015).

In collaboration with the Madison (WI) Police Department, we conducted a pilot study of an MBI similar to MBRT in 30 police officers. First, we sought to demonstrate feasibility, acceptability, and adherence of a modified mindfulness training curriculum in a new police department and geographical region, critical preliminary work for future well-powered randomized trials. Second, we attempted to replicate previously reported improvements in perceived stress, sleep quality, depression, anxiety, and burnout following 8 weeks of mindfulness training (Christopher et al., 2016, 2018). Third, we tested the novel question of whether mindfulness training would lead to reduced symptoms of PTSD in these officers.

\section{Methods}

\section{Participants}

Participants for this study were sworn law enforcement officers employed by the Madison (WI) Police Department (MPD) serving in non-command positions (i.e., patrol officers, officers in non-patrol positions, patrol sergeants, detectives, and investigators). To yield a representative and generalizable sample, the only exclusionary criteria were previous participation in MBSR or similar mindfulness interventions, extensive previous meditation experience, or work schedules that precluded regular participation in mindfulness classes. The primary mode of recruitment was an email sent out to all department staff on behalf of the research team, along with flyers posted at all district stations. The PI also presented information 
about the study at the MPD Officer Advisory Council, and at officer briefings during each major shift change. Interested officers contacted research staff directly, who conducted a brief phone screening interview and scheduled participants for an in-person assessment. We enrolled a total of 30 participants in two cohorts of 15 officers each, with one cohort enrolled in September 2016 and one in February 2017.

Importantly, MPD allowed its officers to take part in classes during work hours or to earn compensatory time for taking part in classes outside of scheduled work hours. Class times were identified jointly with MPD such that they would be minimally disruptive to work schedules, thus facilitating participation by as many officers as possible.

\section{Assessments.}

In-person pre-training (T1) and post-training (T2) assessments were held in private rooms at the UW Hospital Clinical Research Unit in the 2 weeks prior to the first class (mean $\pm \mathrm{SD}=$ $7.8 \pm 4.8$ days, range $=1-16)$ and the 3 weeks following the final class (mean $\pm \mathrm{SD}=11.8 \pm 5.8$ days, range $=1-21)$. At the first visit participants completed informed consent, and participants received monetary compensation for all research activities, which took place while officers were not working.

Each visit began with the application of peripheral psychophysiological sensors, consisting of skin conductance and EKG electrodes and an abdominal breath belt, and psychophysiological data were collected using a BIOPAC MP-150 system during a 5-minute resting baseline and each of 2 behavioral tasks. These tasks included the Affective Go/No-Go task (Hare, Tottenham, Davidson, Glover, \& Casey, 2005), which assesses the impact of socialemotional stimuli on inhibitory control; and the Breath Count task (Levinson, Stoll, Kindy, Merry, \& Davidson, 2014), a measure of sustained attention to the breath and mind-wandering that has been put forth as a putative behavioral indicator of mindfulness. Psychophysiological 
and behavioral data were collected as exploratory measures that could address hypothesized mechanisms of change following mindfulness training. In the interest of space, details on psychophysiological and behavioral data analysis and results are not presented here.

Following behavioral data collection, electrodes were removed and participants moved to a second room where self-report and physical data were collected. Physical measures obtained by a nurse included height, weight, hip-to-waist ratio, blood pressure, pulse, and a blood draw used to assay high-sensitivity C-reactive protein (hs-CRP), a marker of peripheral inflammation that shows reductions in some mindfulness interventions (Black \& Slavich, 2016).

Self-report assessments included multiple measures obtained in a previous pilot study of MBRT in police officers (Christopher et al., 2016), allowing for a direct replication of previous findings in a separate department and with a modified mindfulness intervention, while including several novel outcomes (indicated with a *). Self-report measures included:

- The Police Stress Questionnaire (PSQ; McCreary and Thompson 2006), which contains separate 20 -item scales assessing perceived stress related to both organizational stressors (example items: "dealing with coworkers", "lack of resources", and "bureaucratic red tape") and operational stressors (example items: "risk of being injured on the job", "working alone at night", and "feeling like you are always on the job") of policing. We adapted the original measure to ask about stress over the past 1 month, rather than the past 6 months.

- The 10-item version of the Perceived Stress Scale (PSS; Cohen and Williamson 1988), which assesses past-month appraisals of general (i.e., not occupationally-specific) stressors and one's ability to cope with these stressors. Unfortunately, incorrect anchor points were displayed for the first cohort which resulted in uninterpretable values, so results for the PSS are not presented here. 
- The Oldenburg Burnout Inventory (OLBI; Halbesleben and Demerouti 2005), a 16-item measure of work burnout with separate subscales for exhaustion and disengagement;

- The Pittsburgh Sleep Quality Inventory (PSQI; Buysse et al. 1989), which contains 10 items assessing subjective sleep quality and sleep disruptions over the past month.

- The PTSD Checklist - Civilian Version* (PCL-C; Weathers et al. 1993), a 17-item measure of DSM-IV PTSD symptoms over the past month for use in the general population with separate scales for re-experiencing, avoidance/numbing, and hyperarousal.

- The Patient Reported Outcomes Measurement Information System (PROMIS®), a NIH Roadmap system that provides reliable, precise, and economical measurement of a wide array of health outcomes. We used the 43-item adult inventory, which assesses depression, anxiety, fatigue, sleep disturbances, ability to participate in social roles and activities, pain interference, pain intensity, and physical function. All measures ask about the past 7 days, other than physical and social function, which have no specified time frame.

- The Work Limitations Questionnaire - Short Form* (WLQ-8; Lerner et al. 2002), which contains 8 items assessing the impact of physical and mental health problems on work performance and productivity, which are weighted to yield a single measure corresponding to percent of at-work productivity loss.

- The 18-item version of the Psychological Well-Being Scale* (PWB; Ryff and Keyes 1995), which assesses 6 dimensions of eudaimonic well-being.

- The Positive and Negative Affect Schedule* (PANAS; Watson, Clark, and Tellegen 1988), a 20-item measure of trait levels of positive and negative affect. 
In addition to these outcomes that were obtained at multiple assessments, at the $\mathrm{T} 1$ visit only we collected basic demographic information and assessed lifetime exposure to 34 distinct types of critical incidents of policing using the Critical Incident History Questionnaire* (CIHQ; Weiss et al. 2010). At the conclusion of the initial visit, participants were provided with a Fitbit Charge HR, which they were asked to wear at all times (unless charging, showering, bathing, or swimming) until the time of their follow-up visit. They were also asked to log their work hours using paper logs throughout the 8-week intervention. These Fitbits were used to assess feasibility, acceptability, and adherence for future research that will investigate heart rate and objective sleep duration as primary outcomes, and data are not presented here.

In a follow-up remote assessment (T3) completed approximately 5 months after the end of the intervention $(157.4 \pm 13.7$ days, range $=140-195)$, participants completed all self-report measures from T1 and T2 other than the WLQ-8, PWB, and PANAS (28/30 participants completed this follow-up). We also administered retrospective questions regarding amount and frequency of practice completed since the end of the intervention.

Participants were invited to take part in semi-structured interviews approximately 6 months after the end of the intervention. These optional interviews, which were completed by 15/30 officers, provided us with qualitative data on the impact of this training on officers' lives and how they were incorporating class content into their lives at work and outside of work. Data analysis is ongoing and results are not presented here.

\section{$\underline{\text { Intervention }}$}

In consultation with the PI, two experienced mindfulness teachers created and co-taught an 8-week, 18-hour mindfulness-based curriculum tailored for law enforcement officers, intended to support officer's resilience and well-being both on the job, in their personal lives, and in all the ways those two are interwoven (see Appendix A for overview). The structure and 
content of this curriculum draws from Mindfulness-Based Stress Reduction (MBSR) and Mindfulness-Based Resilience Training (MBRT; Christopher et al. 2016)). The class content and delivery were also heavily influenced by conversations and interactions with MPD officers during in-services, ride-alongs, and other conversations in the year prior to the classes.

Weekly classes were held at a police training facility for 2 hours each week, other than the week 7 class, which was a 4-hour extended "day of practice" (a structural feature adopted from MBRT). In consultation with the police department, we chose class times that would be minimally disruptive for officers' work schedules and held the 2 classes at different times of day to allow participation by a greater number of officers. Classes consisted of the following aspects: 1) didactic instruction around operational definitions of mindfulness, stress, emotions, and scientific findings related to the practices being explored; 2) mindfulness practices including mindfulness of the breath and body, a body scan, mindful movement (including walking meditation and adapted yoga or tai chi practices), mindfulness of thoughts and emotions, mindful speaking and listening, mindful eating, and compassion practice; and 3) inquiry in dyads, triads, or the full group about participants' experiences in practice.

Participants were provided with guided practices recorded by the instructors and were encouraged to practice regularly (6 days/week) between sessions, starting with around 9 minutes/day and increasing to 20 minutes/day by the end of the training. This is a substantially lower request than other MBIs (including both MBSR and MBRT), but the instructors believed it important for this population to set an attainable target and ask for regular, lighter engagement rather than longer practices that participants would be less likely to complete. In addition, participants were provided with various "informal/integrated" practices or drop-ins that might just last a few moments. Some of these practices simply involved becoming aware of present moment experience; others included practices that manipulated breathing, e.g., "straw breathing" 
or the "4,5,6 breath" (breathing in for a count of 4, holding for a count of 5, exhaling for a count of 6). These informal practices served the purpose of integrating practices into daily professional and personal activities. Using either paper or electronic logs, participants were asked to track the type and duration of formal practices, and the frequency with which they engaged in informal mindfulness practices, on a daily basis.

At the end of each weekly class officers were asked to complete anonymous surveys that included questions about training acceptability. In addition, officers were invited to participate in an optional focus group held the week after the final class. These focus groups, which were moderated by an individual not involved in other study activities, provided us with valuable feedback on the class location, timing, instructors, structure, and content.

Data analysis.

To test for differences in self-report and behavioral measures between assessments, we conducted linear mixed effects analysis of repeated measures data using the lme 4 package in $\mathrm{R}$ version 3.2.2, allowing us to retain participants with missing observations. Each model included fixed effects of Time (T1, T2, T3), Gender, and Years Police Experience and a random effect of Subject with a random intercept and fixed slope, e.g.:

$$
\text { model }=\operatorname{lmer}(\text { Outcome } \sim \text { Time }+ \text { Gender }+ \text { Years Policing }+(1 \mid \text { Subject })
$$

Each model initially included the interaction of Time with Gender and Years Policing, and additional fixed effects of Cohort and Cohort*Time. These interaction terms and the fixed Cohort effect were removed from the model using backwards model selection if they did not account for significant variance in the outcome of interest. For these multiple degree of freedom tests (e.g., an omnibus test to identify any effect of Time, or tests comparing models with and without interaction terms), deviance tests were used through the anova.lmerModLmerTest() function in R, comparing models with and without the fixed effect of interest. 
For outcomes that showed a significant omnibus effect of Time, follow-up $t$-tests of differences between T1-T2 or T1-T3 were conducted. Inferences for these single degree-offreedom tests used Satterthwaite's degree of freedom estimation supplied by the lmerTest library in R (https://www.jstatsoft.org/article/view/v082i13/0).

We tested for changes in practice variables across the 8-week intervention by modeling Time as a linear fixed effect and including a random intercept and random slope by subject, e.g.:

$$
\text { model }=\operatorname{lmer}(\text { Practice } \sim \text { Weeks }+(1+\text { Weeks } \mid \text { Subject })
$$

Data and $\mathrm{R}$ code will be provided by the authors upon reasonable request.

\section{Results}

\section{$\underline{1 .}$ Feasibility, adherence, and acceptability}

1a. Feasibility: Recruitment, screening, and retention outcomes

Across two waves of recruitment, 57 MPD officers (representing approximately $12 \%$ of the sworn officers within the department) responded to recruitment materials and completed phone screening, of whom 49 met inclusion criteria. The other 8 individuals were ineligible due to scheduling conflicts $(n=5)$, job duties $(n=2)$, and prior meditation experience $(n=1)$. From this pool of eligible participants, we enrolled a group of officers of roughly equal gender balance that was generally representative of the department as a whole with regard to job title, shift, geographical district, race/ethnicity, and years of experience (see Table 1 for participant characteristics).

All 30 officers completed the 8-week mindfulness intervention as well as the T1 and T2 assessments, and 29/30 officers completed the online T3 follow-up assessment 5 months later. These data on enrollment and retention demonstrate excellent feasibility of delivering an 8-week mindfulness intervention to police officers in a research context. 


\section{1b. Adherence: Class attendance and practice reporting outcomes}

Adherence to the mindfulness training was uniformly high. Overall class attendance was $85 \%$, with no officers missing more than $3 / 8$ classes, promising statistics that were bolstered by the department allowing officers to take part in classes during work hours.

Officers completed and returned $91 \%$ of weekly practice logs through the 8 -week program. Participants reported engaging in formal practice outside of class on average 43.0/56 days $(\mathrm{SD}=9.3$, range $=22-56)$, with an average of 83.8 total practice sessions $(\mathrm{SD}=34.3$, range $=32-170$ occasions $)$ and 870 average minutes of formal practice $(S D=417$, range $=342-2140)$. This is equivalent to nearly 2 hours of weekly formal, out-of-class practice over the 8 -week period. As shown in Figure 1a, linear mixed effects modeling of formal practice time showed a linear increase between weeks 1-8 $(t(29.9)=2.24, p=0.033)$, with a high of 143 minutes in week 7 before falling off in week 8 (when no specific practices were assigned).

We also tracked "informal" practice or moments of mindfulness, reflecting the extent to which participants are integrating practices into activities of daily living. Participants reported engaging in some kind of informal mindfulness practice on average $43.2 / 56$ days $(\mathrm{SD}=12.6$, range $=10-56$ ), and across the training estimated an average of 179 individual instances of informal practice $(\mathrm{SD}=107$, range $=31-497)$. There was a significant linear increase in informal mindfulness practice occasions over the 8 -week intervention $(t(30.0)=2.59, p=0.015$; Figure 1b), with an increase from 17.3 occasions in week 1 to a high of 29.1 occasions in week 7 .

Interestingly, the between-subject correlation between weekly formal practice time and estimated instances of informal practice was non-significant $(r(28)=0.21, p=0.26$; Figure 1c), suggesting that officers' proclivity to engage in formal mindfulness practices was uncorrelated with the frequency that they reported engaging in less formal mindfulness practices in daily living. 
At a T3 assessment completed online approximately 5 months after the classes ended, $79 \%$ of participants reported they had continued formal practice at least on a weekly basis since the conclusion of the intervention, with an average of 1.8 days/week. The most commonly used practices at follow-up were the body scan (13/28 participants), sitting meditation (10/28 participants), and mindful movement (9/28 participants). $96 \%$ of participants reported engaging in informal practice or moments of mindful awareness at least on a daily basis, with participants equally likely to report using such informal practice at work and outside of work.

Collectively, these data on attendance and practice suggest excellent adherence to the intervention requirements, with frequent use of both formal and informal practice techniques during the intervention period and at least some sustained practice engagement in most participants up to 5 months after the end of the intervention.

1c. Acceptability: Study procedures; Intervention content, practices, and instructors At the $\mathrm{T} 2$ visit participants rated different aspects of the assessment location (the UW Hospital Clinical Research Unit, or CRU) and procedures. Participants uniformly reported they felt "in good hands at the CRU" (mean rating 5.0/5.0), were comfortable with the physical procedures carried out by the nurse (4.9/5.0), and were comfortable taking part in this visit in a large, public location (4.4/5.0). Participants had the opportunity to provide open-ended feedback about the behavioral measures, self-report measures, and physical procedures, which did not reveal any areas of concern with regard to acceptability.

Feedback on the Fitbits was generally less positive. A number of participants found it difficult to charge devices or upload data over an 8-week period, with 50\% reporting moderateto-severe annoyance with this aspect of the study and 30\% of participants reported moderate-tosevere discomfort. This feedback suggests that researchers considering the incorporation of 
activity monitors should limit the data collection period and collect feedback on participant comfort and acceptability in pilot testing.

Regarding intervention acceptability, at the conclusion of each of the 8 weekly classes teachers distributed anonymous feedback forms and asked participants to rate the following questions on a 5-point Likert scale from "disagree" to "agree":

- "What we did in class was relevant to my life"

- "I am clear about my mindfulness practice for the upcoming week"

- "I am becoming more familiar with mindfulness practice"

- "I am safe enough in class to explore mindfulness practice"

- "I felt understood by the teachers"

In weeks 1-2 there were between 1-3 participants who rated one or more of these statements as "neutral" or "somewhat disagree". However, beginning in week 3, participants universally rated each of these statements as "moderately agree" or "agree", indicating excellent acceptability for the class content, practices, context, and instructors.

2. Stress-related physical and mental health outcomes

2a. Effects on perceived job stress and burnout

The impact of 8 weeks of mindfulness training on organizational and operational stress was moderated by officers' gender and years of police experience, respectively. For perceived organizational stress, decomposition of a significant Time*Gender interaction $\left(\chi^{2}(2, \mathrm{~N}=9)=7.23\right.$, $p=0.027)$ suggested a steeper decline in stress for male vs. female officers at $\mathrm{T} 2(t(58.2)=2.59$, $p=0.012)$ and at $\mathrm{T} 3(t(58.4)=2.13, p=0.038$; Figure 2a). For perceived operational stress, which showed a significant Time*Years of Experience interaction $\left(\chi^{2}(2, \mathrm{~N}=9)=7.40, p=0.025\right)$, less experienced officers showed relatively greater declines in stress at T2 $(t(58.2)=2.01, p=$ $0.049)$ and T3 $(t(58.4)=2.70, p=0.0092)($ Figure 2b). For scores on both scales, sustained (and, 
in fact, enhanced) effects were observed at 5-month follow-up, at which time effect sizes were comparable to those observed in Christopher et al. (2016) (Table 2).

Reduced burnout was observed for the Exhaustion $\left(\chi^{2}(2, \mathrm{~N}=7)=9.88, p=0.0072\right)$ but not the Disengagement subscale $\left(\chi^{2}(2, \mathrm{~N}=7)=1.66, p=0.44\right)$ of the Oldenburg Burnout Inventory. Significant reductions in Exhaustion were seen at both T2 $(t(58.2)=-3.20, p=0.0023)$ and T3 $(t(58.2)=-2.21, p=0.031)$.

\section{2b. Effects on mental health and well-being}

Consistent with previous research (Christopher et al., 2016), mindfulness training had a large and significant effect on anxiety symptoms on the PROMIS $\left(\chi^{2}(2, \mathrm{~N}=7)=22.97, p<0.001\right.$; $\mathrm{T} 2 t(57.6)=-4.56, p<0.001 ; \mathrm{T} 3 t(58.0)=-4.59, p<0.001)$. A trend reduction was observed for PROMIS depression symptoms $\left(\chi^{2}(2, \mathrm{~N}=7)=5.64, p=0.060\right)$. Increased psychological wellbeing was seen at T2 $(t(30)=-2.19, p=0.036)$, as was decreased trait negative affect on the PANAS $(t(30)=-2.92, p=0.0066)$, although there was no change in trait positive affect $(t(30)=$ $0.81, p=0.42$; each of these measures was obtained at $\mathrm{T} 1$ and $\mathrm{T} 2$ only).

\section{2c. Effects on sleep and physical health}

Consistent with previous research (Christopher et al., 2016), mindfulness training resulted in improved sleep quality as measured by the Pittsburgh Sleep Quality Inventory $\left(\chi^{2}(2\right.$, $\mathrm{N}=7)=11.98, p=0.0025)$. This improvement was significant at T2 $(t(56.4)=-3.65, p<0.001)$ and trending at T3 $(t(56.4)=-1.78, p=0.082)$, although it was not mirrored by improvements in the fatigue or sleep disturbances scales of the PROMIS $\left(\chi^{2}(2, \mathrm{~N}=7)=3.18, p=0.20 ; \chi^{2}(2, \mathrm{~N}=7)=\right.$ $1.14, p=0.57)$. In contrast to findings from Christopher and colleagues $(2016)$, there were no effects of the intervention on PROMIS subscales of pain interference $\left(\chi^{2}(2, \mathrm{~N}=7)=5.80, p=\right.$ $0.055)$, pain intensity $\left(\chi^{2}(2, \mathrm{~N}=7)=4.23, p=0.12\right)$, or physical functioning $\left(\chi^{2}(2, \mathrm{~N}=7)=4.05, p\right.$ 
$=0.13$ ). We also saw no change in the impact of health symptoms on the ability to carry out one's work, as measured by the Work Limitations Questionnaire $(t(30)=1.0, p=0.00)$.

In addition to self-report data, we measured several physical health outcomes at $\mathrm{T} 1$ and $\mathrm{T} 2$ visits only. There were no intervention effects for any of these outcomes, which included logtransformed high-sensitivity C-reactive protein, diastolic and systolic blood pressure, and resting pulse rate (all $t \mathrm{~s}<1.0$, all $p \mathrm{~s}>0.4)$.

\section{Symptoms of posttraumatic stress disorder}

3a. Reduced PTSD symptoms following mindfulness training

Prior to mindfulness training, officers reported low-to-moderate levels of PTSD symptoms on the PCL (mean $\pm \mathrm{SD}=29.9 \pm 6.8$ ), with 12/30 meeting a liberal suggested cut-off for sub-threshold PTSD (33 or higher; Dickstein et al. 2015). Although only 1 participant met DSM-IV diagnostic criteria for all 3 symptom clusters, many participants met criteria for hyperarousal and re-experiencing symptoms (37\% and 53\%, respectively), with relatively few participants meeting criteria for avoidance symptoms (only 13\%). The officer who met all diagnostic criteria had a total PCL score of 61, which was greater than 3 SD greater than the sample mean, and was excluded from further analyses (results were similar when including this officer).

Mindfulness training resulted in lower total PTSD symptoms on the PCL $\left(\chi^{2}(2, N=7)=\right.$ $12.65, p=0.0018)$, an effect that was observed immediately following the training $(t(56.1)=$ 3.55, $p<0.001)$ and also at 5-month follow-up $(t(56.7)=-2.82, p=0.0066$; Figure 3a).

Investigation of individual symptom clusters revealed a significant effect of Time for reexperiencing symptoms $\left(\chi^{2}(2, \mathrm{~N}=7)=9.74, p=0.0077\right)$, which showed no change at T2 $(t(56.2)$ $=-1.35, p=0.18)$ yet showed a significant and large reduction at $\mathrm{T} 3(t(56.9)=-3.26, p=0.0019$; Figure 3b). No effects were seen for avoidance symptoms $\left(\chi^{2}(2, \mathrm{~N}=7)=2.18, p=0.34\right.$; Figure 
3c). For hyperarousal symptoms, a main effect of Time was qualified by a significant Time*Cohort interaction $\left(\chi^{2}(2, \mathrm{~N}=10)=7.74, p=0.021\right.$; Figure 3d $)$. Decomposition of this interaction revealed a reduction in symptoms for both cohorts at T2 (cohort 1: $t(14)=-2.70, p=$ 0.017 ; cohort 2: $t(13)=-5.55, p<0.001)$, although the second cohort of officers showed a larger reduction and only this cohort sustained symptom reductions at $\mathrm{T} 3$ (cohort $1: t(12)=-0.96, p=$ 0.36 ; cohort 2: $t(13)=-4.37, p<0.001)$.

3b. Reductions in PTSD symptoms are associated with reductions in perceived stress but not with improved sleep.

Previous research in urban police officers has shown stress associated with routine aspects of the work environment to be correlated with PTSD symptoms (Maguen et al., 2009). We replicated this relationship in the current sample at baseline both for perceived organizational $(r(27)=0.55, p=0.002)$ and operational stress $(r(27)=0.42, p=0.02)$. Moreover, we found that reductions in perceived stress were correlated with reductions in PTSD symptoms both at T2 (organizational stress: $r(27)=0.40, p=0.033$; operational stress: $r(27)=0.30, p=0.11$ ) and at T3 (organizational stress: $r(25)=0.35, p=0.078$; operational stress: $r(25)=0.37, p=0.060$ ).

Previous research has also identified an association between PTSD symptoms and impaired sleep (Chopko et al., 2018), which we replicated using baseline total PCL symptoms and sleep impairment measured on the PSQI $(r(27)=0.41, p=0.029)$. Whereas this previous study identified relationships for both avoidance and hyperarousal symptoms, in the current study this relationship was entirely driven by hyperarousal symptoms $(r(27)=0.61, p<0.001$; for both re-experiencing and avoidance, $r(27)=0.17, p=0.37)$. In spite of this relationship at baseline, and in contrast to perceived stress findings, reductions in PTSD symptoms were not correlated with reductions in sleep impairment at T2 (total scores $r(27)=0.16, p=0.41$; 
individual subscales all $r \mathrm{~s}<0.23, p \mathrm{~s}>0.23$ ) or T3 (total scores $r(24)=0.02, p=0.92$; individual subscales all $r \mathrm{~s}<0.19, p \mathrm{~s}>0.36)$.

\section{Discussion}

In this pilot study of 30 Madison (WI) police officers, we investigated an 8-week, mindfulness-based training program intended to provide officers with embodied practices and skills to cope with the daily occupational stressors of policing. We collected extremely promising evidence for the feasibility, acceptability, and adherence of the training program and associated research procedures. We observed improvements in self-reported work stress, sleep quality, and stress-related mental health outcomes, replicating previous findings for a similar training program in Oregon police officers. We also collected the first evidence that a mindfulness-based intervention can reduce PTSD symptoms in police officers. This pilot study provides promising

preliminary data for future well-powered, randomized trials that can establish robust evidence for treatment efficacy and mechanisms of action.

Following up on prior work with officers in the metro Portland (OR) area (Christopher et al., 2016, 2018), we sought to establish feasibility, acceptability, and protocol adherence for a modified 8-week mindfulness training program and research procedures in a new police department and metropolitan region. We were successful in recruiting and retaining our entire sample of 30 participants through the 8 -week course and Time 2 assessment and lost only 1 participant at follow-up online testing 5 months later. These participants were highly engaged in the training, attending $85 \%$ of classes, returning $91 \%$ of weekly practice logs, and completing an average of 109 minutes of formal mindfulness practice outside of weekly classes. Participants also reported frequent engagement in "informal" practices and moments of mindful awareness throughout the 8-week training period, demonstrating that they were successfully taking these 
practices "off the cushion" and integrating them into their daily lives. There are two key factors that we believe accounted for this high level of participation and engagement.

First, the training was co-delivered by two teachers who were not only expert mindfulness instructors, but who invested an extensive amount of time and effort before and during the study period gaining "cultural competency" around policing and learning about the stress associated with this profession. The instructors went on ride-alongs with officers, presented at in-service trainings, met extensively with officers on the training team, became familiar with the specific culture and history of this particular police department, and modified the practices and class content in response to these experiences and participant feedback. The impact of these efforts was reflected in near-universal feedback from participants that the class material was relevant to their lives, that they felt understood by teachers, and that they felt safe and comfortable engaging in practices that might be considered "weird" or "touchy-feely" if delivered without consideration of cultural and occupational factors specific to policing.

Second, these efforts by the instructors were accompanied by explicit statements of support by the police department and concrete actions that facilitated study participation by its officers. Participation was fully voluntary but also outwardly supported by the Chief of Police and others in leadership roles, front-line supervisors, and, critically, the police union. We worked with the department to identify class times that would facilitate participation with minimal impact on staffing and participants' home lives, and we delivered the training at a police facility to provide officers with a sense of safety and familiarity. Officers were allowed to participate in classes during work hours whenever possible and received compensatory time when this was not feasible. Through explicit statements of support and concrete actions that facilitated participation, the department underscored its commitment to officer well-being and ensured the success of this pilot study. 
We found that 8 weeks of mindfulness training led to significant reductions in perceived stress for stressors associated with policing, as well as the emotional exhaustion aspect of burnout. Perceived organizational stress showed a steep decline specifically for male officers, whereas perceived operational stress declined over time for less experienced officers in particular. The interaction with years of experience for operational stress may be partially attributable to the fact that less experienced officers are more likely to work in less desirous positions and work shifts and to face greater exposure to operational stressors, and thus have greater room for improvement. It is unclear why mindfulness training would lead to a greater reduction in perceived organizational stress for male vs. female police officers. Additional research in larger samples is needed to illuminate this unexpected result - and, more generally, to understand differential stressor exposure and its impact for men vs. women in policing, a vastly understudied area (Hartley, Mnatsakanova, Burchfiel, \& Violanti, 2014).

We replicated some but not all of the previously reported improvements in stress-related health outcomes noted by Christopher and colleagues (2016) in their previous pilot study of MBRT in Hillsboro (OR) police officers. We observed a significant improvement in subjective sleep quality on the PSQI immediately after the training, but (in contrast to this previous study) no changes on PROMIS scales of fatigue or sleep disturbances. We also observed an immediate large magnitude reduction in anxiety symptoms that persisted at 5-month follow-up, but only trend-level improvement in depression symptoms. State negative affect, a trait-like indicator of risk for mood and anxiety disorders, also showed a significant reduction following mindfulness training. We did not find evidence for improvement in any indicators of physical health outcomes, including PROMIS scales of pain intensity, pain interference, and overall physical functioning (each of which showed numeric, though not significant, worsening following mindfulness training). We also did not identify any impact of the training on resting heart rate, 
blood pressure, or serum levels of high-sensitivity C-reactive protein. Future, well-powered studies with an appropriate control group are needed to clarify what impact if any mindfulness training may have on stress-related physical health outcomes for police officers.

We report here the first empirical evidence that a mindfulness-based intervention can reduce symptoms of PTSD in a police population. This finding resonates with a previous correlational study that identified inverse relationships between facets of self-reported trait mindfulness and PTSD symptoms (Chopko \& Schwartz, 2013). Although most participants did not appear to meet diagnostic criteria for PTSD, about 1/3 met a more liberal cut-point of 33 that has previously been used to screen for potential subthreshold PTSD (Dickstein et al., 2015). This level of PTSD symptoms is consistent with that observed in a previous study of 193 officers from small- to mid-sized Midwest police departments (Chopko et al., 2018). This previous study used structural equation modeling to demonstrate an effect of PTSD symptoms on poorer sleep quality, which in turn mediated the relationship between PTSD symptoms and elevated depression and poorer physical health. Although we also identified a correlation at baseline between elevated PTSD symptoms (hyperarousal symptoms in particular) and poorer sleep quality on the PSQI, between-subject correlations between reductions in PTSD symptoms and sleep impairment were not significant.

In contrast, reductions in perceived organizational and operational stress were correlated with reduced PTSD symptoms. Although it may seem intuitive that critical incident exposure should dictate emergence of PTSD in police officers, stress associated with more routine daily work events has been shown to correlate more strongly with PTSD symptoms in experienced police officers (Liberman et al., 2002) and those one year out of the academy (Maguen et al., 2009). Knowing that critical incident exposure is to some extent an intractable aspect of police work, preventative approaches to PTSD in law enforcement should focus on changing one's 
relationship to daily operational and organizational stressors. A fundamental tenet of mindfulness training is that even if external events are uncontrollable, we can exert control over the way in which we respond and relate to these events. Providing officers with greater perceived control over their environment and responses to stressful events may help buffer the impact of firsthand and vicarious trauma, an idea that is supported by the relationship between changes in perceived stress and PTSD symptoms.

Importantly, there were different trajectories of change for different PTSD symptom clusters. Large reductions in hyperarousal were observed immediately after mindfulness training and persisted at 5-month follow-up. At baseline, over half of participants indicated clinically significant levels of hyperarousal symptoms, which include disrupted sleep, distractibility, increased vigilance, and elevated startle responses. This is perhaps unsurprising as police training emphasizes constant "situational awareness" or scanning the external environment for potential sources of threat. This vigilance is adaptive in contexts where the likelihood of threat is high but can be maladaptive when indiscriminately applied in objectively safe, non-work contexts. In contrast to this external vigilance, mindfulness training encourages what one of our class participants referred to as "internal situational awareness", with practices that turn attention inward toward one's breath, body, thoughts, and emotions. These practices may increase officers' awareness of how they are responding in a particular situation and whether that response is appropriate given the current threat level, and also provide tools for regulating elevated arousal and "re-setting" in between challenging calls for at the end of a work shift. Our promising data on reduced hyperarousal will benefit from follow-up studies that identify the specific mechanisms that lead to symptom change, and whether self-reported symptom improvement is mirrored in objective reductions in hypervigilant behavior, sleep improvement, or physiological indices. 
Reductions in re-experiencing were not observed immediately but emerged at 5-month follow-up, whereas there were no changes in avoidance symptoms at either time point. We hesitate to offer what would be purely speculative explanations for the delayed change in reexperiencing symptoms, given the small sample size and the observation that several participants showed unexpected increases at T2. The failure to observe changes in avoidance following mindfulness training may be due to low baseline levels of these symptoms: only $13 \%$ of participants met DSM-IV criteria for these symptoms at baseline, vs. 53\% and $37 \%$ for hyperarousal and re-experiencing symptoms, respectively. Furthermore, a particular challenge for assessing avoidance of internal experiences such as difficult thoughts, emotions, or memories - especially when using self-report measures - is that avoidant individuals may be especially unlikely to acknowledge or even recognize their tendency to avoid engaging with such experiences. Despite the absence of effects for mindfulness on avoidance symptoms, it remains of interest to continue investigating the potential impact of this training on these symptoms in larger samples and perhaps using alternative means of assessing experiential avoidance, such as interview techniques or behavioral assays. Training in mindfulness may provide an antidote to culturally engrained habits of emotional avoidance and suppression (Berking, Meier, \& Wupperman, 2010; Pogrebin \& Poole, 1995), encouraging officers instead to cultivate an attitude of acceptance and non-judgment for traumatic memories and the complex emotions these memories can elicit.

Notably, the effect sizes for reductions in perceived stress and re-experiencing PTSD symptoms were greater at 5-month follow-up than immediately post-intervention, and several other measures (anxiety, exhaustion, PTSD hyperarousal symptoms) also showed sustained improvements at T3 (Table 2). These findings are in contrast to recent findings from Christopher and colleagues (2018) who identified post-training reductions for perceived stress and other 
outcomes for MBRT vs. a wait-list group that were largely absent at their 3-month follow-up. Although differences in populations or the implementation of these trainings could account for this discrepancy in sustained impact, the absence of a control group in the current study is a significant limitation as there may be factors unrelated to the training resulting in lower levels of stress for MPD officers at follow-up testing. Should future research validate the promising selfreport findings from the current study and other work in this area, a clear challenge for researchers and practitioners to take on together is the development of supports and infrastructure to help sustain improvements in well-being beyond the acute aftermath of an intervention.

There are several limitations of this study, which are largely related to the primary aim being the assessment of feasibility and not intervention efficacy. Our experimental design did not include a control group and was not statistically powered to detect anything but large effects. In addition, most of the outcomes reported here are for self-report measures, which can suffer from responder bias, demand characteristics, and initial "elevation bias" (Shrout et al., 2017) that can confound apparent intervention effects in the absence of a control group. Furthermore, we elected to report intervention effects for all outcomes rather than pre-specifying a more limited number of primary outcomes, and we acknowledge the large number of statistical tests that were conducted without any effort to control for multiple comparisons. Finally, while the percentage of female and non-white participants met or exceeded their respective proportions in the police department as a whole, our sample is less representative with regard to work shifts. Specifically, while we experimented with 2 different class times in an attempt to accommodate all schedules, $87 \%$ of participants worked days/evenings and only $13 \%$ worked overnight shifts, meaning we cannot make strong conclusions about intervention efficacy or acceptability for these "night fighters". 
We are currently conducting a follow-up randomized controlled trial in a much larger sample of police officers across different police agencies. This study seeks to establish the efficacy of mindfulness training for a variety of stress-related outcomes not limited to self-report, including hormonal and inflammatory biomarkers, objectively measured sleep and heart rate in the field, and behavioral assays that can speak to potential mechanisms of action. We are hopeful that this ongoing work will serve to more conclusively demonstrate the ways in which mindfulness training may promote more adaptive responses to the many stressors of policing, with consequent benefits for police officer health and - equally importantly - for the benefit of society as a whole. 


\section{References}

Berking, M., Meier, C., \& Wupperman, P. (2010). Enhancing Emotion-Regulation Skills in Police Officers: Results of a Pilot Controlled Study. Behavior Therapy, 41(3), 329-339. https://doi.org/10.1016/j.beth.2009.08.001

Black, D. S., Reilly, G. A. O., Olmstead, R., Breen, E. C., Irwin, M. R., O’Reilly, G. A., ... Irwin, M. R. (2015). Mindfulness meditation and improvement in sleep quality and daytime impairment among older adults with sleep disturbances: A randomized clinical trial. JAMA Internal Medicine, 175(4), 494-501. https://doi.org/10.1001/jamainternmed.2014.8081

Black, D. S., \& Slavich, G. M. (2016). Mindfulness meditation and the immune system: A systematic review of randomized controlled trials. Annals of the New York Academy of Sciences. https://doi.org/10.1111/nyas.12998

Brown, J., \& Campbell, E. (1990). Sources of occupational stress in the police. Work \& Stress, 4(4), 305-318. https://doi.org/10.1080/02678379008256993

Buysse, D. J., Reynolds, C. F., Monk, T. H., Berman, S. R., \& Kupfer, D. J. (1989). The Pittsburgh sleep quality index: A new instrument for psychiatric practice and research. Psychiatry Research, 28(2), 193-213. https://doi.org/10.1016/0165-1781(89)90047-4

Chopko, B. A., Palmieri, P. A., \& Adams, R. E. (2018). Trauma-Related Sleep Problems and Associated Health Outcomes in Police Officers: A Path Analysis. Journal of Interpersonal Violence, 088626051876791. https://doi.org/10.1177/0886260518767912

Chopko, B. A., \& Schwartz, R. C. (2013). The Relation Between Mindfulness and Posttraumatic Stress Symptoms Among Police Officers. Journal of Loss and Trauma, 18(1), 1-9. https://doi.org/10.1080/15325024.2012.674442

Christopher, M. S., Goerling, R. J., Rogers, B. S., Hunsinger, M., Baron, G., Bergman, A. L., \& Zava, D. T. (2016). A Pilot Study Evaluating the Effectiveness of a Mindfulness-Based 
Intervention on Cortisol Awakening Response and Health Outcomes among Law

Enforcement Officers. Journal of Police and Criminal Psychology, 31(1), 15-28.

https://doi.org/10.1007/s11896-015-9161-x

Christopher, M. S., Hunsinger, M., Goerling, R. J., Bowen, S., Rogers, B. S., Gross, C. R., ... Pruessner, J. C. (2018). Mindfulness-based resilience training to reduce health risk, stress reactivity, and aggression among law enforcement officers: A feasibility and preliminary efficacy trial. Psychiatry Research, 264, 104-115.

https://doi.org/10.1016/j.psychres.2018.03.059

Cohen, S., \& Williamson, G. (1988). Perceived stress in a probability sample of the United States. The Social Psychology of Health. https://doi.org/10.1111/j.15591816.1983.tb02325.x

Dickstein, B. D., Weathers, F. W., Angkaw, A. C., Nievergelt, C. M., Yurgil, K., Nash, W. P., ... Study, R. (2015). Diagnostic Utility of the Posttraumatic Stress Disorder ( PTSD ) Checklist for Identifying Full and Partial PTSD in Active-Duty Military. https://doi.org/10.1177/1073191114548683

Fox, J., Desai, M. M., Britten, K., Lucas, G., Luneau, R., \& Rosenthal, M. S. (2012). Mentalhealth conditions, barriers to care, and productivity loss among officers in an urban police department. Connecticut Medicine, 76(9), 525-531.

Franke, W. D., Collins, S. A., \& Hinz, P. N. (1998). Cardiovascular disease morbidity in an Iowa law enforcement cohort, compared with the general Iowa population. Journal of Occupational and Environmental Medicine, 40(5), 441-444.

https://doi.org/10.1097/00043764-199805000-00006

Galatzer-Levy, I. R., Brown, A. D., Henn-Haase, C., Metzler, T. J., Neylan, T. C., \& Marmar, C. R. (2013). Positive and negative emotion prospectively predict trajectories of resilience and 
distress among high-exposure police officers. Emotion, 13(3), 545-553.

https://doi.org/10.1037/a0031314

Gershon, R. R. M. M., Barocas, B., Canton, A. N., Li, X., \& Vlahov, D. (2009). Mental, physical, and behavioral outcomes associated with perceived work stress in police officers. Criminal Justice and Behavior, 36(3), 275-289. https://doi.org/10.1177/0093854808330015

Goerling, R. J. (2012). Police officer resilience and community building. Proceedings of ASBBS, 19(1), 394-397.

Goyal, M., Singh, S., Sibinga, E. M. S., Gould, N. F., Rowland-Seymour, A., Sharma, R., ... Haythornthwaite, J. A. (2014). Meditation programs for psychological stress and wellbeing: A systematic review and meta-analysis. JAMA Internal Medicine, 174(3), 357-368. https://doi.org/10.1001/jamainternmed.2013.13018

Halbesleben, J. R. B., \& Demerouti, E. (2005). The construct validity of an alternative measure of burnout: Investigating the English translation of the Oldenburg Burnout Inventory. Work \& Stress, 19(3), 208-220. https://doi.org/10.1080/02678370500340728

Hare, T. A., Tottenham, N., Davidson, M. C., Glover, G. H., \& Casey, B. J. (2005). Contributions of amygdala and striatal activity in emotion regulation. Biological Psychiatry, 57(6), 624-632. https://doi.org/10.1016/j.biopsych.2004.12.038

Hartley, T. A., Burchfiel, C. M., Fekedulegn, D., Andrew, M. E., \& Violanti, J. M. (2012). Health disparities in police officers: Comparisons to the U.S. general populations. International Journal of Emergency Mental Health, 13(3), 211-220.

Hartley, T. A., Mnatsakanova, A., Burchfiel, C. M., \& Violanti, J. M. (2014). Stressors and associated health effects for women police officers. In J. M. Violanti (Ed.), Dying for the Job: Police Work Exposure and Health. Springfield, IL: Charles C. Thomas Publisher, Ltd. Khoury, B., Lecomte, T., Fortin, G., Masse, M., Therien, P., Bouchard, V., ... Hofmann, S. G. 
(2013). Mindfulness-based therapy: A comprehensive meta-analysis. Clinical Psychology Review, 33(6), 763-771. https://doi.org/10.1016/j.cpr.2013.05.005

Khoury, B., Sharma, M., Rush, S. E., \& Fournier, C. (2015). Mindfulness-based stress reduction for healthy individuals: A meta-analysis. Journal of Psychosomatic Research, 78(6), 519528. https://doi.org/10.1016/j.jpsychores.2015.03.009

Lerner, D., Amick, B., Rogers, W., Malspeis, S., Bungay, K., Cynn, D., \& Amick. (2002). The Work Limitations Questionnaire 1. Medical Care, 39(1), 72-85. https://doi.org/10.1097/00005650-200101000-00009

Levinson, D. B., Stoll, E. L., Kindy, S. D., Merry, H. L., \& Davidson, R. J. (2014). A mind you can count on: Validating breath counting as a behavioral measure of mindfulness. Frontiers in Psychology, 5, 1-10. https://doi.org/10.3389/fpsyg.2014.01202

Liberman, A. M., Best, S. R., Metzler, T. J., Fagan, J. A., \& Weiss, D. S. (2002). Routine occupational stress and psychological distress in police. Policing: An International Journal of Police Strategies \& Management, 25(2), 421-441. https://doi.org/http://dx.doi.org/10.1108/13639510210429437

Maguen, S., Metzler, T. J., McCaslin, S. E., Inslicht, S. S., Henn-Haase, C., Neylan, T. C., \& Marmar, C. R. (2009). Routine work environment stress and PTSD symptoms in police officers. J Nerv Ment Dis, 197(10), 754-760. https://doi.org/10.1097/NMD.0b013e3181b975f8

McCreary, D. R., \& Thompson, M. M. (2006). Development of two reliable and valid measures of stressors in policing: The operational and organizational police stress questionnaires. International Journal of Stress Management, 13(4), 494-518. https://doi.org/10.1037/10725245.13 .4 .494

Neylan, T. C., Metzler, T. J., Best, S. R., Weiss, D. S., Fagan, J. A., Liberman, A. M., ... 
Marmar, C. R. (2002). Critical incident exposure and sleep quality in police officers. Psychosomatic Medicine, 64(2), 345-352. https://doi.org/10.1097/00006842-20020300000019

Patterson, G. T., Chung, I. W., \& Swan, P. G. (2012). The effects of stress management interventions among police officers and recruits. Campbell Systematic Reviews, 7. https://doi.org/10.4073/csr.2012.7

Pogrebin, M. R., \& Poole, E. D. (1995). Emotion management: A study of police response to tragic events. - PsycNET. Social Perspectives on Emotion, 3, 149-168. Retrieved from https://psycnet.apa.org/record/1997-36435-007

Polusny, M. A., Erbes, C. R., Thuras, P., Moran, A., Lamberty, G. J., Collins, R. C., ... Lim, K. O. (2015). Mindfulness-based stress reduction for posttraumatic stress disorder among veterans a randomized clinical trial. JAMA - Journal of the American Medical Association, 314(5), 456-465. https://doi.org/10.1001/jama.2015.8361

Rajaratnam, S. M. W., Barger, L. K., Lockley, S. W., Shea, S. A., Wang, W., Landrigan, C. P., ... Czeisler, C. A. (2011). Sleep Disorders, Health, and Safety in Police Officers. JAMA, 306(23), 2567. https://doi.org/10.1001/jama.2011.1851

Ramey, S. L., Downing, N. R., \& Franke, W. D. (2009). Milwaukee Police Department retirees: Cardiovascular disease risk and morbidity among aging law enforcement officers. $A A O H N$ Journal, 57(11), 448-453. https://doi.org/10.3928/08910162-20091019-02

Ryff, C. D., \& Keyes, C. L. (1995). The structure of psychological well-being revisited. Journal of Personality and Social Psychology, 69(4), 719-727. Retrieved from http://www.ncbi.nlm.nih.gov/pubmed/7473027

Shrout, P. E., Stadler, G., Lane, S. P., McClure, M. J., Jackson, G., Clavel, F., ... Bolger, N. (2017). Initial elevation bias in subjective reports. Proceedings of the National Academy of 
Sciences. https://doi.org/10.1073/pnas.1712277115

Surawy, C., Roberts, J., \& Silver, A. (2005). The Effect of Mindfulness Training on Mood and Measures of Fatigue, Activity, and Quality of Life in Patients with Chronic Fatigue Syndrome on a Hospital Waiting List: A Series of Exploratory Studies. Behavioural and Cognitive Psychotherapy, 33(1), 103-109. https://doi.org/10.1017/S135246580400181X

Violanti, J. M., Burchfiel, C. M., Miller, D. B., Andrew, M. E., Dorn, J., Wactawski-Wende, J., ... Trevisan, M. (2006). The Buffalo Cardio-Metabolic Occupational Police Stress (BCOPS) Pilot Study: Methods and Participant Characteristics. Annals of Epidemiology, 16(2), 148-156. https://doi.org/10.1016/j.annepidem.2005.07.054

Watson, D., Clark, L. A., \& Tellegen, A. (1988). Development and validation of brief measures of positive and negative affect: The PANAS scales. Journal of Personality and Social Psychology, 54(6), 1063-1070. https://doi.org/10.1037/0022-3514.54.6.1063

Weathers, F., Litz, B., Herman, D., Huska, J., \& Keane, T. (1993). The PTSD Checklist (PCL): Reliability, validity, and diagnostic utility. In The Annual Convention of the International Society for Traumatic Stress Studies. San Antonio, TX.

Weiss, D. S., Brunet, A., Best, S. R., Metzler, T. J., Fagan, J. A., \& Marmar, C. R. (2010). Frequency and severity approaches to indexing exposure to trauma: The Critical Incident History Questionnaire for police officers. Journal of Traumatic Stress, 23(6), 734-743. https://doi.org/10.1002/jts.

Zimmerman, F. H. (2012). Cardiovascular disease and risk factors in law enforcement personnel: A comprehensive review. Cardiology in Review, 20(4), 159-166. https://doi.org/10.1097/CRD.0b013e318248d631 
Tables

\begin{tabular}{|c|c|c|}
\hline Characteristic & $\mathbf{N}$ & $\%$ \\
\hline \multicolumn{3}{|l|}{ Gender } \\
\hline Female & 16 & $53 \%$ \\
\hline Male & 14 & $47 \%$ \\
\hline \multicolumn{3}{|l|}{ Race } \\
\hline Caucasian & 26 & $87 \%$ \\
\hline Asian & 1 & $3 \%$ \\
\hline Black & 1 & $3 \%$ \\
\hline American Indian & 1 & $3 \%$ \\
\hline Other/Multiple & 1 & $3 \%$ \\
\hline \multicolumn{3}{|l|}{ Ethnicity } \\
\hline Hispanic or Latinx & 5 & $17 \%$ \\
\hline Not Hispanic or Latinx & 25 & $83 \%$ \\
\hline \multicolumn{3}{|l|}{ Work Detail } \\
\hline $1^{\text {st }}(\sim 0600-1400)$ & 12 & $40 \%$ \\
\hline $2^{\text {nd }}(\sim 1200-0800)$ & 5 & $17 \%$ \\
\hline $3^{\text {rd }}(\sim 1400-2200)$ & 9 & $30 \%$ \\
\hline $4^{\text {th }}(\sim 2000-0400)$ & 0 & $0 \%$ \\
\hline $5^{\text {th }}(\sim 2200-0600)$ & 4 & $13 \%$ \\
\hline \multicolumn{3}{|l|}{$\begin{array}{l}\text { Rank/job } \\
\text { responsibilities }\end{array}$} \\
\hline Sergeant & 3 & $10 \%$ \\
\hline Detective & 2 & $7 \%$ \\
\hline Investigator & 1 & $3 \%$ \\
\hline Officer in patrol & 16 & $53 \%$ \\
\hline $\begin{array}{r}\text { Officer, non-patrol } \\
\text { role }\end{array}$ & 8 & $27 \%$ \\
\hline \multicolumn{3}{|l|}{ Marital Status } \\
\hline Married & 18 & $60 \%$ \\
\hline $\begin{array}{l}\text { Unmarried } \\
\text { relationship }\end{array}$ & 7 & $23 \%$ \\
\hline Single & 5 & $17 \%$ \\
\hline \multirow[t]{2}{*}{ Divorced } & 0 & $0 \%$ \\
\hline & Mean & St. Dev. \\
\hline Age & 38.4 & 7.7 \\
\hline $\begin{array}{l}\text { Years police } \\
\text { experience }\end{array}$ & 10.8 & 7.5 \\
\hline
\end{tabular}

Table 1. Participant demographics and work information 


\begin{tabular}{|c|c|c|c|c|c|c|c|c|c|c|}
\hline Measure & $\begin{array}{c}\text { Time } 1 \\
\text { Mean (SD) }\end{array}$ & $\begin{array}{c}\text { Time } 2 \\
\text { Mean (SD) }\end{array}$ & $\begin{array}{c}\text { Time } 3 \\
\text { Mean (SD) }\end{array}$ & $\begin{array}{l}\text { Interactions } \\
\qquad\left(\chi^{2}, p\right)\end{array}$ & $\begin{array}{c}\text { Main Effect } \\
\text { of Time }\left(\chi^{2},\right. \\
p)\end{array}$ & $\begin{array}{l}\text { T2-T1 } \\
(t, p)\end{array}$ & $\begin{array}{l}\text { T3-T1 } \\
(t, p)\end{array}$ & T2-T1 d & T3-T1 d & $\begin{array}{l}\text { Christopher } \\
\text { et al. (2016) d }\end{array}$ \\
\hline \multicolumn{11}{|l|}{ Police Stress Q'airre } \\
\hline Organizational Stress & $66.6(11.3)$ & $60.8(10.5)$ & $54.0(9.9)$ & $\begin{array}{l}\text { Time* Gender } \\
(7.23, .027)\end{array}$ & $\cdot$ & . & 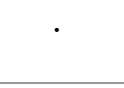 & -0.37 & -0.76 & 0.72 \\
\hline Operational Stress & $67.1(12.0)$ & $60.6(10.4)$ & $54.4(12.0)$ & $\begin{array}{l}\text { Time*Years } \\
(7.40, .025)\end{array}$ & . & . & - & -0.42 & -0.66 & 0.56 \\
\hline $\begin{array}{l}\text { Oldenburg Burnout } \\
\text { Total }\end{array}$ & $39.4(3.9)$ & $37.1(3.2)$ & $38.0(3.5)$ & & $5.92, .052$ & $\cdot$ & & -0.44 & -0.22 & 0.74 \\
\hline Exhaustion & $20.2(2.0)$ & $18.6(1.8)$ & $19.0(2.0)$ & & $9.88, .0072$ & $\begin{array}{l}-3.20 \\
.0023\end{array}$ & $\begin{array}{l}-2.21 \\
.031\end{array}$ & -0.59 & -0.36 & 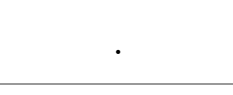 \\
\hline Disengagement & $19.2(2.4)$ & $18.5(2.0)$ & $19.0(2.1)$ & & $1.66, .44$ & & & -0.22 & -0.05 & . \\
\hline PSQI & $7.6(1.7)$ & $5.9(1.5)$ & $6.5(1.9)$ & & $11.98, .0025$ & $\begin{array}{l}-3.65 \\
<.001\end{array}$ & $\begin{array}{c}-1.77 \\
.082\end{array}$ & -0.73 & -0.28 & \\
\hline PROMIS Scales & & & & & & & & $\cdot$ & · & $\begin{array}{l}0.48(\mathrm{PH}) / \\
0.78(\mathrm{MH})^{2}\end{array}$ \\
\hline Anxiety & $12.0(2.3)$ & $9.4(2.1)$ & $9.3(2.3)$ & & $22.97,<.001$ & $\begin{array}{l}-4.56 \\
<.001\end{array}$ & $\begin{array}{l}-4.59 \\
<.001\end{array}$ & -0.86 & -0.76 & \\
\hline Depression & $9.3(2.2)$ & $8.1(1.4)$ & $8.5(2.2)$ & & $5.41, .067$ & . & . & -0.48 & -0.29 & \\
\hline Fatigue & $15.5(3.4)$ & $14.0(3.4)$ & $14.6(3.2)$ & & $3.18, .20$ & . & . & -0.31 & -0.20 & 0.59 \\
\hline Sleep Disturbances & $14.8(3.1)$ & $14.1(3.5)$ & $15.0(3.5)$ & & $1.14, .57$ & . & . & -0.15 & -0.05 & 0.74 \\
\hline Pain Intensity & $2.5(1.1)$ & $3.0(1.2)$ & $3.0(0.9)$ & & $4.23, .12$ & . & . & $0.30^{3}$ & $0.38^{3}$ & \\
\hline Pain Interference & $7.5(2.6)$ & $8.9(2.7)$ & $8.9(2.0)$ & & $5.8, .055$ & . & . & $0.35^{3}$ & $0.42^{3}$ & 0.37 \\
\hline Physical Functioning & $29.2(1.0)$ & $29.1(0.8)$ & $28.7(1.0)$ & & $4.05, .13$ & . & . & $0.10^{3}$ & $0.33^{3}$ & . \\
\hline Social Participation & $15.5(4.2)$ & $14.0(3.1)$ & $13.9(3.2)$ & & $3.46, .18$ & & & $0.28^{3}$ & $0.22^{3}$ & \\
\hline PTSD Checklist Total & $29.9(4.5)$ & $25.7(4.9)$ & $26.2(3.9)$ & & $12.65, .0018$ & $\begin{array}{l}-3.55 \\
<.001\end{array}$ & $\begin{array}{l}-2.82 \\
.0066\end{array}$ & -0.58 & -0.54 & \\
\hline Re-experiencing & $8.0(1.7)$ & $7.3(2.2)$ & $6.4(1.7)$ & & $9.74, .0077$ & $\begin{array}{c}-1.35 \\
.18\end{array}$ & $\begin{array}{l}-3.26 \\
.0019\end{array}$ & -0.23 & -0.76 & . \\
\hline Avoidance & $10.9(2.5)$ & $9.9(2.4)$ & $10.4(2.7)$ & & $2.18, .34$ & . & . & -0.28 & -0.03 & . \\
\hline Hyperarousal & $11.0(1.6)$ & $8.5(1.7)$ & $9.4(1.2)$ & $\begin{array}{l}\text { Time*Cohort } \\
(7.74, .021)\end{array}$ & . & · & - & -1.01 & -0.70 & \\
\hline WLQ-8 ${ }^{1}$ & $3.1(2.0)$ & $3.1(2.0)$ & . & & 0,1 & . & . & 0.00 & . & . \\
\hline Psychol. Well-Being ${ }^{1}$ & $88.2(5.0)$ & $90.9(5.0)$ & $\cdot$ & & $4.45, .035$ & $\begin{array}{l}-2.19 \\
.036\end{array}$ & - & -0.39 & $\cdot$ & . \\
\hline \multicolumn{11}{|l|}{ PANAS $^{1}$} \\
\hline Trait Positive Affect & $36.8(3.7)$ & $37.6(2.7)$ & . & & $0.65, .42$ & . & . & -0.15 & . & . \\
\hline Trait Negative Affect & $18.8(2.7)$ & $16.9(2.7)$ & . & & 7.49, .0062 & $\begin{array}{l}-2.92 \\
.0066\end{array}$ & & -0.52 & . & \\
\hline
\end{tabular}


Table 2. Summary of self-report effects. Mean values for each self-report measure obtained from participants at Time 1 (baseline), Time 2 (immediately post-mindfulness training), and T3 (3-month follow-up). Significant interactions between Time and Gender, Years of Experience, and Cohort (class 1/class 2) are reported, and in the absence of such interactions we report the omnibus test of Time. For a significant omnibus effect of time, we indicate results of paired $t$ tests comparing scores between T1 and both T2/T3. Regardless of statistical significance, effect sizes for each measure between T1-T2 and T1-T3 are provided, along with analogous T1-T2 effect sizes for a previous pilot study of a similar 8-week mindfulness intervention for police officers (Christopher et al., 2016). Bolded entries represent statistically significant effects at an uncorrected threshold of $p<$ 0.05. Abbreviations: PSQI = Pittsburgh Sleep Quality Inventory; PROMIS = Patient Reported Outcomes Measurement Information System (43-item inventory); WLQ-8 = Work Limitations Questionnaire, 8-item version; PANAS = Positive and Negative Affective System.

Notes. 1. Indicates measure was not obtained at the follow-up (T3) assessment. 2. Values from Christopher et al. (2016) are for brief composite measures of physical health and mental health, respectively. 3. Effect sizes indicate worsening of this symptom relative to baseline. 


\section{Figure Legends}

Figure 1: Engagement in weekly formal and informal practice. (A) Participants completed an average of 109 minutes of weekly formal practice outside of class, with a significant linear increase from Week 1-8 of the intervention $(t(29.9)=2.24, p=0.033)$. (B) Participants estimated taking part in informal mindfulness practice on average 22 times/week, with a significant linear increase from Week 1-8 $(t(30.0)=2.59, p=0.015)$. (C) There was no significant relationship between weekly time spent in formal mindfulness practice and self-reported occasions of informal practice $(r(28)=0.21, p=0.26)$.

Figure 2: Effects of mindfulness training on perceived organizational and operational stress.

(A) For scores on the Organizational Police Stress Questionnaire, there was a significant Time*Gender interaction, driven by a steeper decline in stress for men vs. women at T2 $(t(58.2)=$ $2.59, p=0.012)$ and at $\mathrm{T} 3(t(58.4)=2.13, p=0.038)$. (B) For scores on the Operational Police Stress Questionnaire, a significant Time*Years of Experience interaction reflected greater declines in stress for relatively less experienced officers at both T2 $(t(58.2)=2.01, p=0.049)$ and $\mathrm{T} 3(t(58.4)=2.70, p=0.0092)$.

Figure 3: Effects of mindfulness training on PTSD symptoms. (A) Total scores on the PTSD Checklist $(\mathrm{PCL})$ showed a significant decline from baseline at T2 $(t(56.1)=-3.55, p<0.001)$ and $\mathrm{T} 3(t(56.7)=-2.82, p=0.0066)$. (B) PTSD re-experiencing symptoms showed a significant decrease at T3 only $(t(56.9)=-3.26, p=0.0019$; T2: $t(56.2)=-1.35, p=0.18)$. (C) There was no significant effect of mindfulness training on PTSD avoidance symptoms. (D) There was a significant Time*Cohort interaction for PTSD hyperarousal symptoms, with a significant decrease from baseline at T2 for both cohorts (cohort 1: $t(14)=-2.70, p=0.017$; cohort $2: t(13)$ $=-5.55, p<0.001)$ and a sustained decrease at T3 for cohort 2 only (cohort $1: t(12)=-0.96, p=$ 0.36; cohort 2: $t(13)=-4.37, p<0.001)$. 
A B C
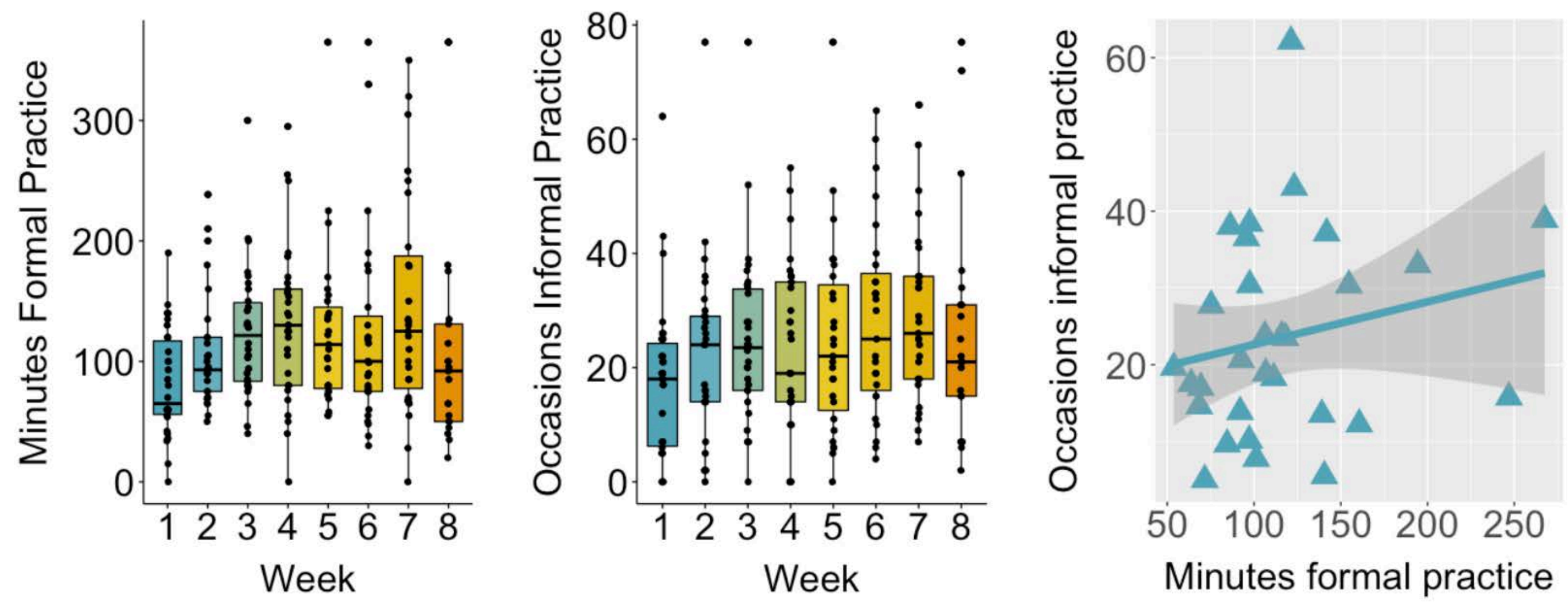
A

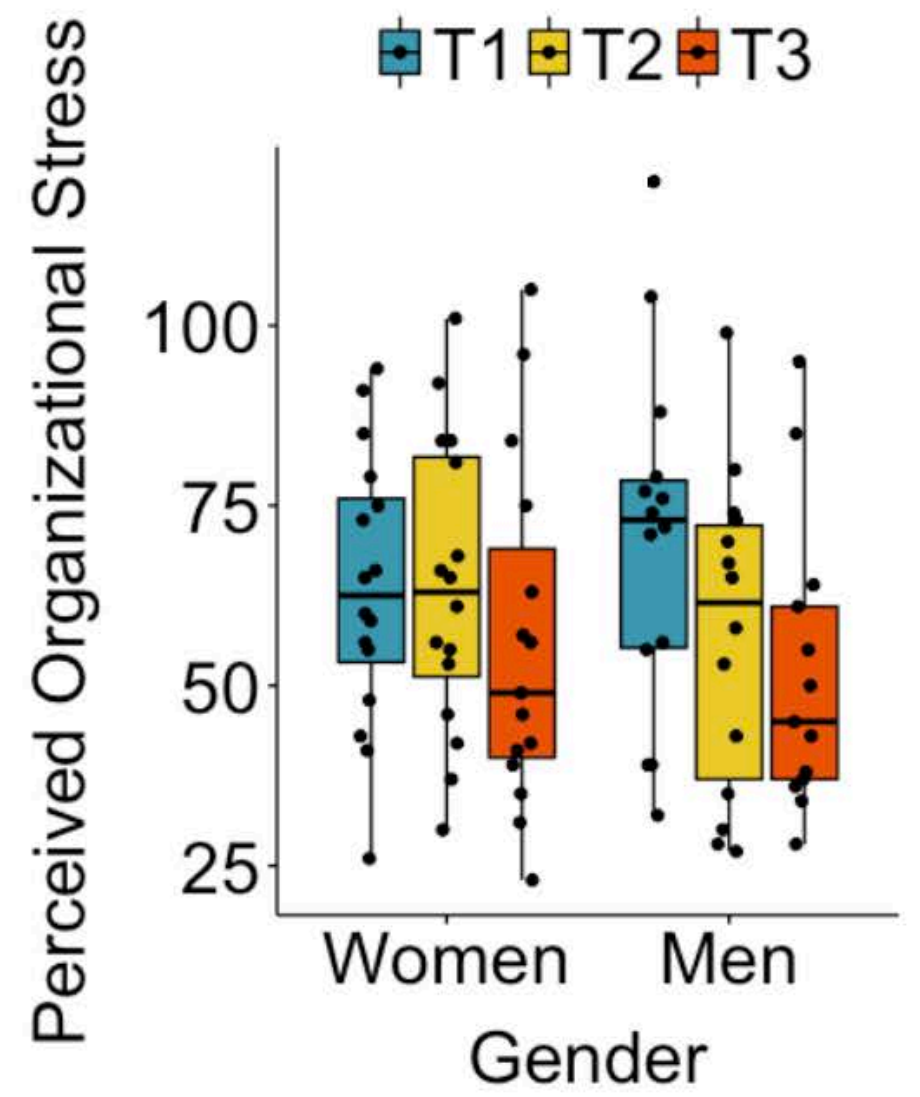

B

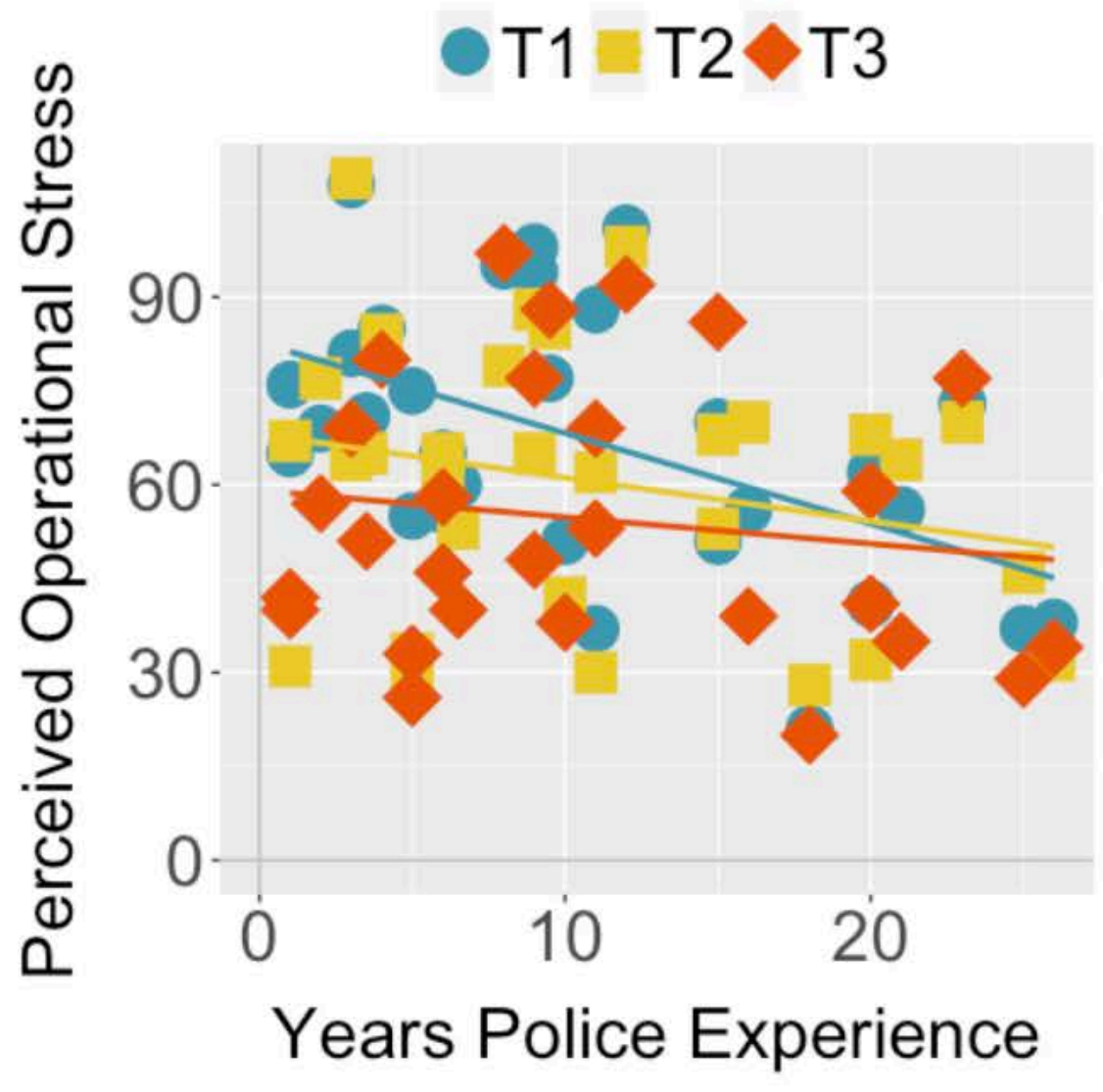


A

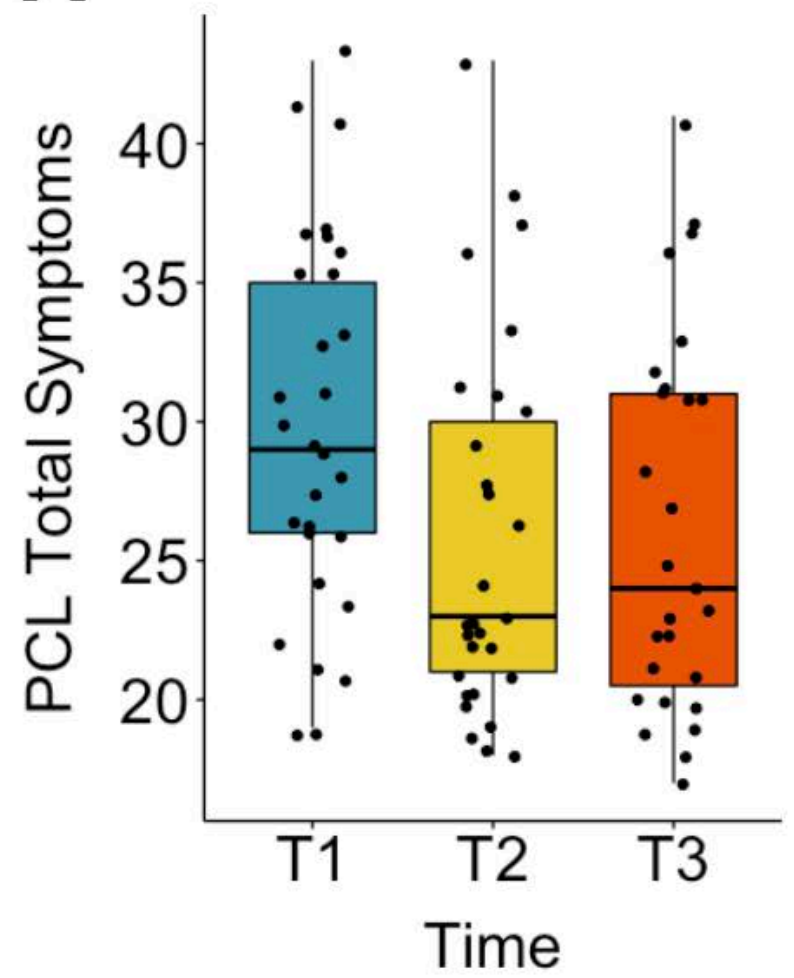

C

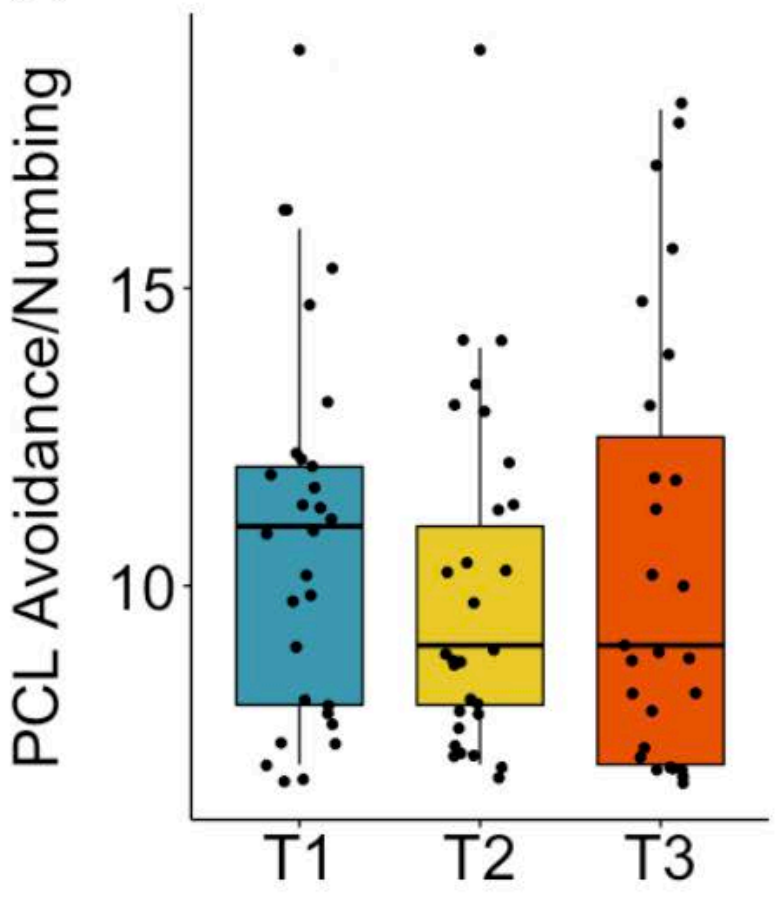

Time
B

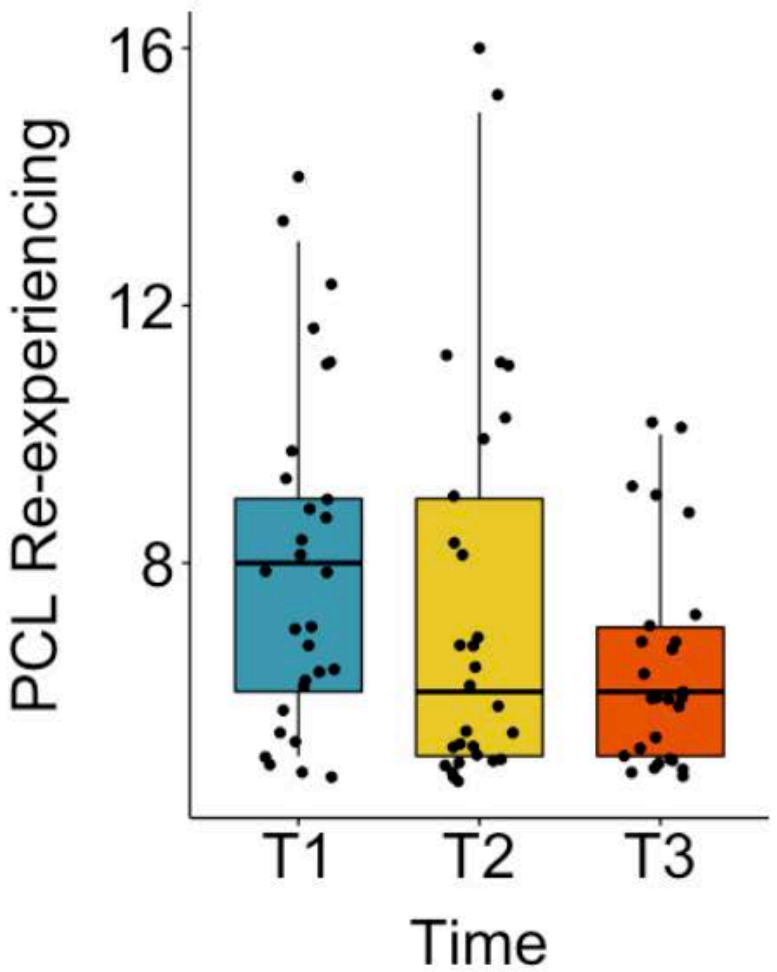

D

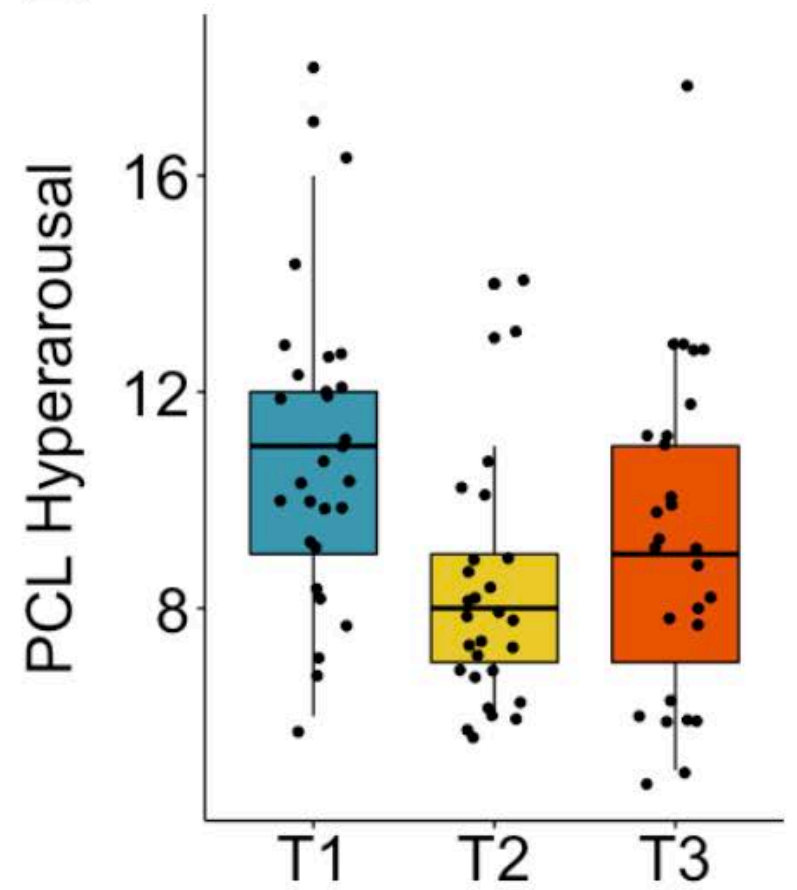

Time 


\section{Appendix A}

\section{Intervention Summary: First Responder Resilience and Mindfulness training}

Overview of 8-week curriculum developed between July 2016-June 2017. This curriculum was inspired by the Mindfulness-Based Resilience Training curriculum created by Mike Christopher, Rich Goerling, and Brant Rogers at Pacific University. We are deeply grateful to them for sharing their wisdom and experience with us.

Week 1: Introduction to Resilience and Mindfulness

Content: Introduction of course/instructors/volunteer/participants. Definition of mindfulness, neuroplasticity and how we can foster greater resilience. Creation of community agreements and discussion of culture. Movement and body scan practices. Debriefing of session 1 practices.

Homework: Explanation of practice log. Formal practice: 9 minutes movement/9 minutes body scan 6 out of 7 days. Informal practice: "Dropping in" and 3 good breaths.

Week 2: Settling into Resilience and Mindfulness

Content: Normalizing mind wandering and addressing other (mis)conceptions about doing the practices "correctly." Movement, body scan and debrief. Introduce walking practice.

Homework: Practice log. Formal practice: Alternate 9 minutes movement/9 minutes body scan 6 out of 7 days. 5 minutes of walking practice 3 times. Informal practice: "Dropping in" and 3 good breaths.

Week 3: Meeting our experiences through Resilience and Mindfulness

Content: Movement, exploration of physical posture, introduction to breath awareness practice and debrief. Reading/video to reconnect to inspiration/motivation.

Homework: Practice log. Formal practice: Alternate 9 minutes of movement $/ 5$ minutes of awareness of breath practice 6 out of 7 days a week. Informal practice: Pleasant Events Calendar, integrated walking practice and 3 good breaths.

Week 4: Meeting Stress with Resilience and Mindfulness

Content: Movement, breath awareness practice and debrief. Introduction of mindful eating.

Homework: Practice Log. Formal practice: Alternate 15 minutes of movement with 15 minutes of a breath awareness practice. Informal practice: Integrated walking practice, Unpleasant Events Calendar and 3 good breaths. 
Week 5: Reactivity, Resilience and Mindfulness

Content: Movement, breath awareness and debrief. Reflection of practice and course at halfway point and resetting of intentions for the remaining 4 weeks. Introduction to Compassion practice.

Homework: Practice Log. Alternate movement/breath awareness practice with compassion practice 6 days out of 7 . Informal practice: Walking practice (1x per week) and 3 good breaths.

Week 6: Response, Resilience and Mindfulness

Content: Movement, awareness of breath practice, compassion practices and debriefing. Discussion and preparation for the Day of Extended Practice.

Homework: Practice log. Alternate between 15 minutes of awareness of breath and compassion practice. Informal practice: Walking practice (1x per week), and 3 good breaths.

Week 7: Extended Practice Session (4 hours)

Content: This session includes all of the previous practices of mMBRT along with mountain meditation and a period of silence.

Homework: Practice log. Participant decides his/her practice.

Week 8: Resilience and Mindfulness: Beginning Again

Content: Movement, breath awareness practice and debriefing. Debriefing about course. Implementation exercise. Review of resources and support for participants.

Homework: Practice Log. Participant decides her/his practice. 